\title{
Problemática psicosocial y de salud mental reportada por consultantes del servicio de atención telefónica Línea de la Vida, derivados a Centros de Integración Juvenil durante la epidemia de COVID-I9
}

\author{
David Bruno Diaz-Negrete (D)', Solveig E. Rodríguez-Kuri (D) ', Cristina de J. Cruz-Cortés (D)', \\ Alma Delia Gutiérrez-López (D)', Juan David González-Sánchez', Carmen Fernández-Cáceres' \\ I Centros de Integración Juvenil, A. C.
}

\section{RESUMEN}

Introducción: en el contexto de la contingencia sanitaria por la pandemia de COVID-19 y por acuerdo adoptado en el Grupo Técnico de Trabajo de Salud Mental de la Secretaría de Salud, Centros de Integración Juvenil colabora en la atención de personas que consultan el servicio Línea de la Vida de la Comisión Nacional contra las Adicciones, por algún problema de salud mental. Objetivo: caracterizar aspectos de la problemática psicológica y social asociados a la epidemia e identificar variables intervinientes. Método: estudio transversal, comparativo, con una muestra de 2,403 consultantes del servicio Línea de la Vida, derivados a ClJ entre mayo y agosto de 2020. La información incluyó síntomas y condiciones de riesgo de COVID-19, problemas de salud mental y circunstancias cotidianas durante la epidemia. Se efectuaron análisis comparativos por sexo, edad y fecha de consulta. Resultados: una tercera parte refirió síntomas de coronavirus, pertenecer a un grupo vulnerable o tener contacto con personas que padecían la enfermedad. Los principales problemas de salud mental fueron ansiedad/estrés (67.6\%) y depresión (15.2\%); 31.5\% refirió problemas económicos, 15.5\%, la pérdida de personas cercanas, cerca de $10 \%$, problemas en el cuidado de niños, adolescentes y otros familiares, y $5.6 \%$, violencia doméstica. Conclusiones: los problemas de salud, sociales, interpersonales y de salud mental asociados a la pandemia reflejan diferencias atribuibles a la construcción social del género, el ciclo de vida de las personas y el desarrollo mismo de la epidemia.

Palabras clave: COVID-19, salud mental, servicios de salud mental, sistemas de apoyo psicosocial.

\begin{abstract}
Introduction: in the context of the health contingency by COVID-19 pandemic and by an agreement of the Mexican Ministry of Health, Centros de Integración Juvenil has collaborated in Línea de la Vida telephone service conducted by the National Comission on Addictions in the care of people with mental health dissorders. Objective: to describe some aspects of the psychological and social problems associated with the epidemic, identifying intervening variables. Method: cross-sectional, comparative study, with a sample of 2,403 consultants from Línea de la Vida service, referred to ClJ between May and August 2020. Data included symptoms and risk conditions of COVID-19, mental health problems and daily circumstances during the epidemic. Comparative analysis were carried out by sex, age and date of consultation. Results: a third of the consultants reported symptoms of COVID-19, belonging to a vulnerable group or having contact with someone with the disease. The main mental health problems identified were anxiety/ stress (67.6\%) and depression (15.2\%); 31.5\% reported facing financial problems; $15.5 \%$ the loss of close people and around $10 \%$ having problems in the care of children, adolescents or other relatives; $5.6 \%$ reported domestic violence. Conclusions: the health, social, interpersonal and mental health problems associated with the COVID-19 pandemic reflect differences attributable to the social construction of gender, the life cycle of people and the development of the epidemic itself.
\end{abstract}

Keywords: COVID-19, mental health, mental health services, psychosocial support systems.

Este trabajo se publica en virtud del interés que puede representar para el conocimiento de los efectos de la crisis sanitaria originada por la epidemia de COVID-19, a pesar de que el tema del uso de drogas ocupa en él un sitio relativamente menor. También refleja la colaboración de Centros de Integración Juvenil, casa editora de esta Revista Internacional de Investigación en Adicciones, en el desarrollo de una respuesta institucional pronta y efectiva a la problemática emergente durante la epidemia.

\footnotetext{
Autor de correspondencia:

C.P. 01900. Correo electrónico: dir.invens@cij.gob.mx

David Bruno Diaz-Negrete. Av. San Jerónimo 372, col. Jardines de Pedregal, alcaldía Álvaro Obregón, Ciudad de México,

Recibido: 8 de octubre de $2020 \quad$ Aceptado: 21 de octubre de 2020 doi: 10.28931/riiad.2020.2.06 


\section{INTRODUCCIÓN}

Con motivo de la contingencia sanitaria ocasionada por la pandemia de COVID-19, las autoridades sanitarias mexicanas pusieron a disposición de la población a nivel nacional el servicio telefónico de asistencia psicológica Línea de la Vida. Creada para atender problemas de abuso de sustancias y de adicciones, bajo la coordinación de la Comisión Nacional contra las Adicciones (CONADIC), durante la pandemia la Línea de la Vida amplió su cobertura para dar respuesta no sólo a problemas por uso de sustancias, sino en general, a problemas de salud mental con incidencia durante la emergencia sanitaria. A partir de marzo de 2020, el Grupo Técnico de Trabajo de Salud Mental de la Secretaría de Salud ajustó sus estrategias para dar respuesta a las solicitudes de atención recibidas a través de este medio, a fin de dar cabida a la colaboración de instituciones y organizaciones que ofrecieron su apoyo para atender una demanda que mostraba un incremento exponencial. Entre estas se encontraba Centros de Integración Juvenil (CIJ), institución dedicada al tratamiento, prevención e investigación del uso de drogas. $\mathrm{ClJ}$ se sumó a la atención de las llamadas telefónicas a través de 148 operadores profesionales que fueron previamente capacitados de manera virtual, mediante los cursos "Todo sobre la prevención del COVID-19", "Entrenamiento continuo sobre manejo clínico para profesionales de la salud", "Plan de acción para el hogar ante el COVID-19" y "Primeros auxilios psicológicos".

En este contexto surge el interés del presente estudio, cuyo objetivo es conocer los principales problemas de salud mental, incluyendo el uso de sustancias, entre la población solicitante del servicio de Línea de la Vida derivada a ClJ; determinar si existen diferencias significativas con respecto a los problemas reportados, atribuibles al género, la edad y al momento de la epidemia en que se solicitó la atención; así como identificar variables intervinientes en la problemática de salud mental observada.

El impacto psicosocial y en la salud mental de enfermedades epidémicas como el ébola, la influenza H1N1 y el síndrome respiratorio severo agudo, así como del aislamiento y del confinamiento como medidas adoptadas para hacerles frente, han sido estudiados con pacientes hospitalizados, profesionales de la salud, residentes en casas de asistencia social (Abad et al., 2010; Huremović, 2019; Johal, 2009; McMahon et al., 2016) e incluso segmentos de la población expuestos a un contagio potencial (Digiovanni et al., 2004; O'Leary, et al., 2018).

En general, los hallazgos indican una alta prevalencia de trastornos del estado de ánimo, depresión, ira y ansiedad, trastornos por estrés postraumático, abuso de sustancias y conductas evitativas (Abad et al., 2010;
Brooks et al., 2020; Day et al., 2013; Hawryluck et al., 2004; Huremović, 2019). En otros casos se reporta malestar atribuible a una situación de incertidumbre y de temor que se ve acentuada por la falta de información y de comunicación que suele ser común al inicio de un brote (Johal, 2009). Igualmente, en una reciente revisión bibliográfica, Brooks y cols. (2020) señalan que las personas en cuarentena presentan un alto temor de ser contagiados o de contagiar a otros, principalmente a otros miembros de su familia, así como sentimientos de aburrimiento, frustración y aislamiento asociados a la pérdida de rutinas y a la reducción de contactos interpersonales, también frustración, ansiedad e ira vinculadas con las dificultades económicas y con un menor acceso a bienes y servicios.

Otro factor relevante, en el caso de grupos específicos, como el caso de los profesionales de la salud o grupos que por alguna característica particular presentan condiciones de alto riesgo, es el estigma social, el cual gravita negativamente sobre las condiciones psicológicas y sociales de las personas, generando pérdida de confianza, rechazo y agresión (O'Leary et al., 2018; McMahon et al., 2016). Igualmente, en el contexto del brote de síndrome respiratorio severo agudo en Toronto, Canadá, en 2003, se encontró que la disposición a respetar las medidas de confinamiento se relaciona con la legitimidad de su adopción, con la claridad de criterios y la credibilidad de la información disponible; igualmente con la seguridad de contar con un ingreso económico y acceso a bienes y servicios; del mismo modo, con la desarticulación del estigma y el rechazo social, así como con la tolerancia hacia quienes por su situación no podían sujetarse al confinamiento y, por último, con la disponibilidad de alternativas para hacer frente al aislamiento y al malestar psicológico (Digiovanni et al., 2004).

En el caso de la epidemia del coronavirus SARSCoV-2, hay un amplio número de estudios que dan cuenta de sus consecuencias en la salud mental, desde síntomas ligeros hasta trastornos graves que pueden afectar profundamente la vida de las personas, tales como depresión, ansiedad o estrés postraumático (Bruine de Bruin, 2020; Durankuş \& Aksu, 2020; Elbay et al., 2020; Fitzpatrick et al., 2020; Huarcaya-Victoria, 2020; Hyland et al., 2020; Johnson et al., 2020; Jungmann \& Witthöft, 2020; Karaşar \& Canli, 2020; Luo et al., 2020; Martínez, 2020; Mazza, De Lorenzo et al., 2020; Mental Health America, 2020; Active Minds, 2020; Mosheva et al., 2020; Ni et al., 2020; Ozamiz-Etxebarria et al., 2020; Özdin \& Bayrak Özdin, 2020; Pappa et al., 2020; Qiu et al., 2020; Ramírez-Ortiz et al., 2020; Rajkumar, 2020; Rehman et al., 2020; Salari et al., 2020; Savitsky et al., 2020; Tsamakis et al., 2020; Torales et al., 2020; Verma \& Mishra, 2020; Wong et al., 2020). 
Los trastornos reportados más frecuentemente en el mundo son ansiedad y depresión, encontrándose tasas más altas de ansiedad entre la población de adultos jóvenes, mujeres, sectores económicamente vulnerables (Elbay et al., 2020; Hyland et al., 2020; Lin et al., 2020; Mental Health America, 2020; Qiu et al., 2020; Özdin \& Bayrak Özdin, 2020; Wong et al., 2020), pacientes con un diagnóstico psiquiátrico previo (Mazza, De Lorenzo, et al., 2020) y personal de salud involucrado directa o indirectamente con la atención de población afectada por la epidemia (Luo et al., 2020; Mosheva et al., 2020; Torales et al., 2020; Savitsky et al., 2020). Por el contrario, destaca que adultos mayores, uno de los grupos más afectados en términos de mortalidad atribuible a COVID-19, muestran, según Bruine de Bruin (2020), niveles menores de depresión y ansiedad que adultos jóvenes.

El trastorno por ansiedad parece estar relacionado, entre otras causas, con la información al alcance de las personas. En este sentido, se ha encontrado que cuanto mayor sea la información acerca de la pandemia de la que se dispone, mayor es la ansiedad (Qiu et al., 2020). De igual modo, se ha observado que la búsqueda excesiva de información en línea se asocia con altos niveles de ansiedad (Jungmann \& Witthöft, 2020; Karaşar \& Canli, 2020; Ni et al., 2020). También se reportan como causas frecuentes de ansiedad, el temor o susceptibilidad percibida al contagio (Lin et al., 2020; Ozamiz-Etxebarria, et al., 2020) y el encierro o el aislamiento forzados o autoimpuestos (Ozamiz-Etxebarria et al., 2020). Otro aspecto asociado es habitar en núcleos urbanos con una alta prevalencia de contagio, de tal modo que es posible encontrar notorias diferencias regionales dentro de un mismo país o territorio (Fitzpatrick et al., 2020; Li et al., 2020; Ni et al., 2020; Özdin \& Bayrak Özdin, 2020).

Atención especial merece la población que ha enfermado de COVID-19 o que tiene algún familiar que ha padecido la enfermedad. Quienes han sobrevivido a ella presentan en muy alta proporción trastornos de estrés postraumático, depresión y ansiedad, así como síntomas obsesivo-compulsivos e insomnio. De acuerdo con Mazza y cols. (Mazza, De Lorenzo et al., 2020) $56 \%$ registra puntuaciones dentro de un rango patológico en al menos alguna de estas categorías clínicas, siendo más alta la puntuación en las mujeres y en los casos con un diagnóstico psiquiátrico previo. De igual manera, los familiares de pacientes con COVID-19 presentan niveles de ansiedad significativamente mayores que el resto de la población (Özdin \& Bayrak Özdin, 2020).

Por otro lado, aunque los efectos de la pandemia muestran importantes coincidencias a nivel global respecto a la presencia de trastornos de ansiedad y depresión, se pueden observar algunos resultados contrastantes, posiblemente atribuibles a diferencias culturales. De este modo, en la India se han observado bajos niveles de estrés y depresión, así como niveles moderados de ansiedad (Rehman et al., 2020).

Otros múltiples estudios reportan un incremento de trastornos depresivos relacionados con la pandemia. Se ha referido tanto un aumento de episodios de depresión mayor (Torales et al., 2020) como el riesgo de suicidio entre quienes han padecido la enfermedad ( $\mathrm{Li}$ et al., 2020). De manera similar a lo que se ha reportado respecto a los trastornos de ansiedad, se han encontrado niveles más altos de depresión en mujeres (Mazza, De Lorenzo et al., 2020; Bruine de Bruin, 2020; Pappa et al., 2020), en habitantes de grandes urbes, en personas con familiares que han sido diagnosticados con la enfermedad (Ni et al., 2020), y entre quienes presentan antecedentes de padecimientos psiquiátricos y enfermedades crónicas (Özdin \& Bayrak Özdin, 2020).

Entre los sectores con mayor riesgo de sufrir trastornos depresivos se encuentra el personal de salud, en particular, las enfermeras (Elbay et al., 2020). Del mismo modo, la presencia de trastornos depresivos registra una mayor asociación con ser mujer, ser joven, tener una menor experiencia laboral y trabajar en la primera línea de atención (Ni et al., 2020). También se ha reportado un incremento de casos de depresión postparto durante la pandemia (Durankuş \& Aksu, 2020). Y, al igual que con la ansiedad, Bruine de Bruin (2020) reporta que entre la población estadounidense ser adulto mayor se asocia con menores niveles de depresión.

También, el aislamiento social (Karaşar, et al., 2020) y una situación laboral y económica inestable (Rehman et al., 2020) son factores asociados con la presencia de trastornos depresivos durante la epidemia. Asimismo, la depresión que sobreviene entre personas contagiadas o entre familiares de quienes han enfermado resulta particularmente alta; en este sentido, Mazza, De Lorenzo y cols. (2020) encontraron en población sobreviviente de COVID-19 cerca de 30\% de casos con depresión en un nivel patológico hasta un mes después de haber obtenido el alta.

Por otra parte, la reducción de la movilidad y el confinamiento de grandes masas de población, recomendadas para contener la ola de contagios en el mundo (Organización Mundial de la Salud [OMS], 2019), han favorecido el aislamiento y la violencia contra las mujeres. A nivel mundial, se estima que $30 \%$ de las mujeres han sufrido violencia de pareja, en la que se incluyen agresiones físicas, sexuales, psicológicas y económicas (Anurudran et al., 2020; Gulati \& Kelly, 2020; Mazza, Marano et al., 2020; Roesch et al., 2020; Sacco et al., 2020). Se ha identificado que las restricciones a la movilidad social y el confinamiento favorecen el contacto sostenido de las mujeres con sus agresores, la inhabilitación 
de redes y mecanismos de apoyo, así como la reducción del alcance de los servicios sociales y asistenciales (Organización de Estados Americanos-Comisión Interamericana de Mujeres [OEA-CIM], 2020; Roesch et al., 2020; Sacco et al., 2020). En el mismo contexto, se ha reportado la emergencia de nuevas formas de violencia contra las mujeres, como la restricción del acceso a productos sanitarios como agua, jabón y desinfectantes, a los servicios de salud por posible contagio y a medicamentos (Anurudran et al., 2020; Gulati \& Kelly, 2020). Las mujeres migrantes, adultas mayores, con alguna discapacidad, en condiciones de crisis humanitaria o pertenecientes a alguna minoría podrían verse más afectadas (OEA-CIM, 2020).

Se ha referido, además, un agravamiento de los problemas familiares y de pareja por la pérdida súbita de medios de vida, la restricción de actividades económicas y laborales, la inseguridad financiera y el abuso de sustancias (Cluver et al., 2020; Humphreys et al., 2020). A esto se suma que los padres y, en particular, las madres se han visto en la necesidad de responder simultáneamente a requerimientos laborales y a las necesidades de cuidado de sus hijos debido al cierre de centros de cuidado infantil y escuelas, el cual ha dejado a los menores no sólo sin acceso a servicios educativos y de cuidado, sino también frente a la posibilidad de verse expuestos a la violencia o a la negligencia familiar sin que se cuente con los medios habituales para la denuncia (Cluver et al., 2020; Humphreys et al., 2020; Mazza, Marano et al., 2020, Sacco et al., 2020). La condición de las mujeres se ve agravada debido a que, en general, suelen ser depositarias de las distintas formas de cuidado informal, entendido como la prestación de cuidado a personas en situación de dependencia, incluyendo el ya señalado cuidado de niños y adolescentes, así como el de enfermos, adultos mayores y personas con discapacidad (Cantillo et al., 2018; Jiménez \& Moya, 2017). Estas tareas, que no son debidamente valoradas ni social ni económicamente, suelen recaer sobre las mujeres, aunque haya otros miembros de la familia disponibles para la tarea, dejándolas en condición de mayor vulnerabilidad (OEA-CIM, 2020). En general, lo anterior concuerda con lo observado en anteriores episodios epidémicos, en los cuales las mujeres experimentaron una mayor probabilidad de contagio debido a su papel en la prestación de cuidados familiares, así como en situaciones de desastre en las que se vieron expuestas a mayores tasas de violencia (Del Río-Lozano \& García-Calvente, 2020; Gulati \& Kelly, 2020).

Con base en estos antecedentes, el presente estudio persiguió caracterizar algunos aspectos de la problemática psicológica y social asociada con la epidemia de COVID-19 en el caso de población solicitante de servicios de atención psicológica a distancia, así como identificar algunas posibles variables intervinientes en la conformación de esta problemática.

Respecto a la evolución de la epidemia en México y sus probables efectos en la salud mental, durante el lapso de recolección de datos para el presente estudio debe señalarse un acusado incremento de casos positivos confirmados de 36,327 a 560,164 , mientras que los fallecimientos pasaron de 3,573 a 60,480. A lo largo de todo el periodo, el país se encontraba en la tercera fase epidemiológica de evolución de la epidemia o de "transmisión comunitaria"; al inicio corría la denominada "Jornada Nacional de Sana Distancia", la cual concluyó el 30 de mayo (Gobierno de México, 2020).

\section{MÉTODO}

Los resultados que aquí se reportan provienen de un estudio transversal, comparativo, realizado con población consultante del servicio de la Línea de la Vida, derivada a ClJ por acuerdo adoptado en el Grupo Técnico de Trabajo de Salud Mental de la Secretaría de Salud de México.

\section{Muestra}

Se recuperó información de 2,403 casos mayores de 18 años, derivados a ClJ entre el 11 de mayo y el 23 de agosto de 2020. El 22.1\% de las llamadas provino de residentes de la Ciudad de México y $17.4 \%$ del estado de México; el resto se distribuyó entre las otras 30 entidades federativas del país.

\section{Procedimiento}

Participaron en la atención de los consultantes 148 terapeutas y consejeros de ClJ, con formación en psiquiatría, medicina, psicología, enfermería y trabajo social, y adscritos a 11 unidades de hospitalización.

Los datos fueron recuperados del registro de información efectuado por los propios terapeutas y consejeros en una matriz de datos preparada ex profeso en una hoja de cálculo del programa Excel. La Subdirección de Consulta Externa estuvo a cargo de la coordinación del programa en ClJ; a lo largo del proceso se acudió a reuniones interinstitucionales con los coordinadores de los Servicios de Atención Psiquiátrica y CONADIC, para resolver dudas o problemas en el registro de información. Asimismo, se llevaron a cabo reuniones periódicas de asesoría y retroalimentación con los responsables de las unidades de $\mathrm{ClJ}$ participantes, con el objeto de resolver dudas o problemas que se hubiesen presentado durante la prestación del servicio o en la captura de datos. La construcción de la base de datos estuvo a cargo del Departamento de Hos- 
pitalización de ClJ; la depuración y análisis de datos, de la Subdirección de Investigación.

\section{Instrumento}

La matriz de registro precodificada fue elaborada por el equipo de profesionales de la Comisión Nacional contra las Adicciones responsables de la Línea de la Vida, incluyendo información sociodemográfica y de identificación del consultante, síntomas y condiciones de riesgo de COVID-19, tipo de intervención realizada, diagnóstico presunto de salud mental y datos de referencia de la consulta (institución, fecha, asesor, etc.). En ClJ se agregaron datos de uso de sustancias, una sección de circunstancias de vida durante la crisis sanitaria (cumplimiento del confinamiento, contacto con personas contagiadas y cohabitantes en la vivienda) y una sección de estado de ánimo percibido y problemática interpersonal y social experimentada durante la epidemia. Las categorías diagnósticas fueron establecidas con base en una lista de verificación preestablecida de signos y síntomas característicos de distintos trastornos de salud mental que los terapeutas corroboraban con cada consultante.

Atendiendo a las condiciones de la toma de datos por parte de los terapeutas, es decir, el contexto de una consulta psicológica a distancia, la mayor parte de las variables tuvieron un formato categórico con el objeto de facilitar su registro.

Para efectos de este trabajo se recuperaron datos sociodemográficos, síntomas y condiciones de riesgo de COVID-19, problemas de salud mental identificados, circunstancias durante la pandemia: cohabitación, apego al confinamiento doméstico, estado de ánimo percibido y problemática interpersonal y social.

\section{Análisis}

El análisis de la información comprendió el conteo descriptivo de frecuencias y la comparación de grupos a través de pruebas de diferencia de proporciones (chi cuadrada). La comparación se efectuó en tres etapas que contemplaron la comparación de: 1) hombres y mujeres; 2) grupos de edad (entre 18 y 29 años, entre 30 y 64, y 65 años y más); y 3) cuatro grupos definidos en función de la fecha de la consulta, según cortes de cuatro semanas (excepto el primer lapso que, dada la disponibilidad de datos, sólo comprendió tres semanas): del 11 al 31 de mayo; del primero al 28 de junio, del 29 de junio al 26 de julio; y del 27 de julio al 23 de agosto.

Posteriormente, se efectuó un análisis multivariado de segmentación no paramétrica, mediante un "árbol de decisión", con el objeto de identificar grupos y caracte- rísticas asociados con una más alta prevalencia de ansiedad y estrés, así como los problemas de salud mental más frecuentemente reportados en la muestra de estudio, tomando como variables independientes características sociodemográficas (sexo, grupo de edad y lapso de consulta), uso de sustancias, variables asociadas a la epidemia (síntomas característicos de COVID-19, condiciones de vulnerabilidad, contagio o temor de contagio, contacto con alguna persona enferma y confinamiento doméstico) y problemas enfrentados durante la misma (problemas económicos, dificultades en el cuidado de niños, jóvenes o adolescentes y familiares enfermos, problemas de pareja, pérdida de personas cercanas, violencia doméstica y otros problemas de salud); sin establecer ningún condicionamiento de variables a la segmentación. Considerando la posible prevalencia de síndromes de "depresión ansiosa", se incluyó el diagnóstico presuntivo de depresión como "variable de influencia".

Como método de crecimiento del árbol se adoptó la opción de CHAID exhaustivo que explora todas las posibles divisiones de cada predictor, con una profundidad de tres niveles, un mínimo de 200 casos en nodos filiales y de 100 en nodos parentales. Para determinar la significancia de la división de nodos, se aplicó la prueba de chi cuadrada de Pearson al nivel habitual de .05. Los análisis fueron realizados con el programa SPSS V. 20.

\section{Consideraciones éticas}

Además de solicitarles los datos requeridos en la hoja de cálculo, se invitaba a los consultantes a participar en una encuesta para fines de investigación, aclarando que su participación era absolutamente voluntaria, anónima y confidencial. La no aceptación de la invitación a participar no afectó en ningún momento la recepción íntegra del servicio.

\section{RESULTADOS}

\section{Resultados generales (Tabla I)}

Cerca de la mitad de los consultantes eran hombres y otro tanto, mujeres. La edad promedio fue de 43.1 años $(D E=13.7)$, con una proporción considerablemente más alta de personas adultas, entre 19 y 64 años (76.0\%). En cuanto al lapso en el que se recibieron las llamadas, cerca de una tercera parte fueron recibidas entre el $11 \mathrm{y}$ 31 de mayo, otro tanto, entre el primero y 28 de junio; una cuarta parte, entre el 29 de junio y el 26 de julio; y cerca de $15 \%$, entre el 27 de julio y el 23 de agosto. Cerca de una quinta parte de los consultantes vivía solo(a); poco más de la mitad, con su pareja o cónyuge, y cerca de 
una tercera parte con niños, jóvenes o adolescentes y/o adultos mayores.

Prácticamente una tercera parte de los consultantes refirió, en cada caso, padecer síntomas de COVID-19, pertenecer a un grupo vulnerable o haber tenido contacto con alguien contagiado; asimismo, una quinta parte registró trastornos comórbiles de alto riesgo (diabetes, hipertensión, sobrepeso y obesidad) y $65.5 \%$ reportó haber podido permanecer en casa durante la epidemia.

Los principales problemas de salud mental identificados fueron ansiedad y estrés, depresión y trastornos psicosomáticos. Los trastornos de ansiedad o estrés incluían síntomas como nerviosismo, angustia, temor, aflicción, revivir la experiencia traumática, intranquilidad e insomnio. Proporciones considerablemente menores refirieron abuso de sustancias, conducta violenta o suicida y trastornos psicóticos. De los consultantes que reportaron abuso de sustancias, 15 señalaron el alcohol como droga de mayor impacto, 15 el tabaco y 10 la mariguana.
En cuanto a otros problemas reportados, más de la mitad refirió haberse contagiado o temer haberse contagiado de COVID-19; una tercera parte, enfrentar problemas económicos o no poder satisfacer cabalmente sus necesidades. Cerca de una sexta parte reportó la pérdida de personas cercanas o dificultades en el cuidado de familiares enfermos, con discapacidad o adultos mayores; entre 5 y 10 por ciento enfrentaba dificultades en el cuidado de niños pequeños o para ejercer su autoridad con jóvenes o adolescentes, experimentaba problemas serios de pareja y había sido o temía ser víctima de violencia doméstica. Por último, la mitad de los casos refirió sentirse intranquilo(a) y con temor; una sexta parte expresó sentirse estresado y rebasado por las dificultades y poco menos de 10 por ciento, triste y deprimido 0 , por el contrario, tranquilo y en calma. Menos del 5 por ciento señaló sentirse molesto o de mal humor, confundido, o bien, por el contrario, confiado en salir adelante.

\section{Tabla 1}

Condiciones de vida y problemática psicosocial y de salud mental reportada por consultantes del servicio de atención telefónica Línea de la Vida, derivados a Centros de Integración Juvenil durante la epidemia de COVID-19

\begin{tabular}{|c|c|c|}
\hline & \multicolumn{2}{|c|}{$\begin{array}{l}\text { Muestra general } \\
\qquad(n=2,403)\end{array}$} \\
\hline & Frec. & $\%$ \\
\hline \multicolumn{3}{|l|}{ Sexo } \\
\hline Hombres & 1,173 & 49.5 \\
\hline Mujeres & 1,230 & 50.5 \\
\hline \multicolumn{3}{|l|}{ Edad (Media: 43.1 años; $D E=13.7$ ) } \\
\hline Entre 18 y 29 años (adultos jóvenes) & 398 & 16.5 \\
\hline Entre 30 y 64 años (adultos) & 1,826 & 76.0 \\
\hline 65 años y más (adultos mayores) & 179 & 7.4 \\
\hline \multicolumn{3}{|l|}{ Fecha de consulta } \\
\hline Entre el 11 y el 31 de mayo & 665 & 27.7 \\
\hline Entre el 1 y el 28 de junio & 752 & 31.3 \\
\hline Entre el 29 de junio y el 26 de julio & 632 & 26.3 \\
\hline Entre el 27 de julio y el 23 de agosto & 354 & 14.7 \\
\hline \multicolumn{3}{|l|}{ Cohabitación durante la pandemia } \\
\hline El (la) consultante vive solo/a & 423 & 17.6 \\
\hline Vive con su cónyuge o pareja & 1,252 & 52.1 \\
\hline Vive con hijos jóvenes o adolescentes & 747 & 31.1 \\
\hline Vive con niños & 666 & 27.7 \\
\hline Vice con adultos mayores & 716 & 29.8 \\
\hline \multicolumn{3}{|l|}{ Variables relacionadas con la pandemia } \\
\hline El (la) consultante reporta síntomas de COVID-19 & 711 & 29.6 \\
\hline Pertenece a un grupo vulnerable & 781 & 32.5 \\
\hline
\end{tabular}


Tabla 1

Condiciones de vida y problemática psicosocial y de salud mental reportada por consultantes del servicio de atención telefónica Línea de la Vida, derivados a Centros de Integración Juvenil durante la epidemia de COVID-19 (continuación)

\begin{tabular}{|c|c|c|}
\hline Ha convivido con alguien con COVID-19 & 704 & 29.3 \\
\hline Registra trastornos comórbiles de alto riesgo & 495 & 20.6 \\
\hline Durante la epidemia le ha sido posible en general quedarse en casa & 1,573 & 65.5 \\
\hline \multicolumn{3}{|l|}{ Diagnóstico presuntivo de salud mental } \\
\hline Ansiedad/estrés & 1,625 & 67.6 \\
\hline Depresión & 366 & 15.2 \\
\hline Trastorno psicosomático & 273 & 11.4 \\
\hline Conducta violenta & 65 & 2.7 \\
\hline Conducta suicida & 51 & 2.1 \\
\hline Psicosis & 48 & 2.0 \\
\hline Abuso de sustancias & 76 & 3.2 \\
\hline \multicolumn{3}{|l|}{ Otras problemáticas } \\
\hline Enfrenta problemas económicos o necesidades insatisfechas & 757 & 31.5 \\
\hline Ha tenido dificultades para ejercer su autoridad con jóvenes o adolescentes & 184 & 7.7 \\
\hline Ha experimentado dificultades con el cuidado de niños pequeños & 181 & 7.5 \\
\hline $\begin{array}{l}\text { Ha tenido problemas con el cuidado de familiares enfermos, con discapacidad o } \\
\text { adultos mayores }\end{array}$ & 318 & 13.2 \\
\hline Ha tenido problemas serios de pareja & 191 & 7.9 \\
\hline Ha sufrido la pérdida de una persona cercana & 372 & 15.5 \\
\hline Ha sido o teme ser víctima de violencia en su casa & 134 & 5.6 \\
\hline Se ha contagiado o teme haberse contagiado de COVID-19 & 1,377 & 57.3 \\
\hline \multicolumn{3}{|l|}{ Estado de ánimo en la última semana } \\
\hline Confiado(a) en que las cosas saldrán adelante & 78 & 3.2 \\
\hline Confundido(a) & 110 & 4.6 \\
\hline Estresado(a), rebasado(a) por las dificultades & 388 & 16.1 \\
\hline Intranquilo(a), con temor & 1,204 & 50.1 \\
\hline Molesto(a) y de mal humor & 116 & 4.8 \\
\hline Tranquilo(a), en calma & 225 & 9.4 \\
\hline Triste, deprimido(a) & 228 & 9.5 \\
\hline
\end{tabular}

\section{Comparación hombres-mujeres (Tabla 2)}

Las mujeres presentaron una edad ligeramente mayor que los hombres; también refirieron en una proporción mayor cohabitar con niños o con hijos jóvenes o adolescentes, mientras que los hombres, en contraste, señalaron más frecuentemente vivir solos.

Las mujeres pertenecían en mayor medida a algún grupo vulnerable a COVID-19, en tanto que los hombres reportaron, en una mayor proporción, padecer síntomas de la enfermedad. De igual modo, las mujeres habían podido mantener con mayor frecuencia las medidas de confinamiento.
Entre las mujeres se registró una mayor frecuencia de trastornos de ansiedad o estrés y depresión, en tanto que entre los hombres el abuso de sustancias presentó un porcentaje significativamente más alto. Ellas expresaron, en proporciones significativamente mayores, tener dificultades en el cuidado de niños pequeños y de otros familiares enfermos, con discapacidad o adultos mayores, así como para ejercer su autoridad con jóvenes o adolescentes. Igualmente, refirieron con frecuencia significativamente más alta haber sido o temer ser objeto de violencia doméstica.

Por último, las mujeres refirieron con una frecuencia más alta sentirse estresadas y rebasadas por las dificultades, mientras que los hombres afirmaron en mayor proporción sentirse tranquilos y en calma. 
Tabla 2

Condiciones de vida y problemática psicosocial y de salud mental reportada por consultantes del servicio de atención telefónica Línea de la Vida, derivados a Centros de Integración Juvenil durante la epidemia de COVID-19

Diferencias significativas por sexo

\begin{tabular}{|c|c|c|c|c|c|}
\hline \multirow{3}{*}{ Edad promedio } & \multirow{2}{*}{\multicolumn{2}{|c|}{$\begin{array}{c}\text { Hombres } \\
(n=1,173)\end{array}$}} & \multicolumn{2}{|c|}{$\begin{array}{c}\text { Mujeres } \\
(n=1,230)\end{array}$} & \multirow{3}{*}{$\begin{array}{c}t=-2.38, p=.018 \\
\boldsymbol{X}^{\mathbf{2}}, \boldsymbol{p}(\boldsymbol{g l}=\mathbf{1})\end{array}$} \\
\hline & & & \multicolumn{2}{|c|}{$43.8(D E=13.5)$} & \\
\hline & Frec. & $\%$ & Frec. & $\%$ & \\
\hline \multicolumn{6}{|l|}{ Cohabitación durante la pandemia } \\
\hline El (la) consultante vive solo/a & 227 & 19.4 & 196 & 16.0 & $4.926,<.001$ \\
\hline Vive con hijos jóvenes o adolescentes & 299 & 25.5 & 448 & 36.4 & $33.493,<.001$ \\
\hline Vive con niños & 282 & 24.0 & 384 & 31.2 & $15.444,<.001$ \\
\hline \multicolumn{6}{|l|}{ Variables relacionadas con la pandemia } \\
\hline El (la) consultante reporta síntomas de COVID-19 & 390 & 33.2 & 321 & 26.1 & $14.735,<.001$ \\
\hline Pertenece a un grupo vulnerable & 356 & 30.3 & 425 & 34.5 & 4.836, .028 \\
\hline $\begin{array}{l}\text { Durante la epidemia le ha sido posible en general quedarse } \\
\text { en casa }\end{array}$ & 697 & 59.4 & 876 & 71.3 & $37.656,<.001$ \\
\hline \multicolumn{6}{|l|}{ Diagnóstico presuntivo de salud mental } \\
\hline Ansiedad/estrés & 761 & 64.8 & 864 & 70.2 & $7.895, .005$ \\
\hline Depresión & 146 & 12.4 & 220 & 17.9 & $13.760,<.001$ \\
\hline Abuso de sustancias & 56 & 4.8 & 20 & 1.6 & $19.426,<.001$ \\
\hline \multicolumn{6}{|l|}{ Otras problemáticas } \\
\hline $\begin{array}{l}\text { Ha tenido dificultades para ejercer su autoridad con jóvenes o } \\
\text { adolescentes }\end{array}$ & 60 & 5.1 & 124 & 10.1 & $29.995,<.001$ \\
\hline Ha experimentado dificultades en el cuidado de niños pequeños & 67 & 5.7 & 114 & 9.3 & $10.788, .001$ \\
\hline $\begin{array}{l}\text { Ha tenido problemas en el cuidado de familiares enfermos, } \\
\text { con discapacidad o adultos mayores }\end{array}$ & 126 & 10.8 & 192 & 15.6 & $12.336,<.001$ \\
\hline Ha sido o teme ser víctima de violencia doméstica & 47 & 4.0 & 87 & 7.1 & $10.723, .001$ \\
\hline \multicolumn{6}{|l|}{ Estado de ánimo en la última semana } \\
\hline Estresado(a), rebasado(a) por las dificultades & 160 & 13.6 & 228 & 18.5 & $10.633, .001$ \\
\hline Tranquilo(a), en calma & 133 & 11.3 & 92 & 7.5 & $10.532, .001$ \\
\hline
\end{tabular}

\section{Comparación por grupos de edad (Tabla 3)}

En primer sitio, se aprecia una proporción creciente de mujeres conforme aumenta la edad y, al contrario, un mayor porcentaje de hombres en el grupo de adultos jóvenes. Los adultos mayores refirieron también condiciones de mayor aislamiento social (vivir solos o solas) mientras que, conforme a lo que cabía esperar, el porcentaje de consultantes que vivían con niños pequeños fue más alto en el grupo de jóvenes adultos, y de quienes vivían con adolescentes o jóvenes, en el de adultos.

En segundo lugar, como también cabía esperar, la comparación arrojó que los adultos mayores presentaban porcentajes mayores de condiciones comórbiles de riesgo y vulnerabilidad, mientras que el grupo de adultos jóvenes reportó con mayor frecuencia síntomas de COVID-19. Los consultantes de mayor edad refirieron, igualmente, una mayor posibilidad de mantener el confinamiento durante la epidemia. Tanto los adultos mayores como los más jóvenes registraron porcentajes ligeramente mayores que los adultos entre 30 y 65 años, de conductas violentas y suicidas, así como abuso de sustancias.

Por último, las dificultades en el cuidado de niños pequeños fueron mayores conforme la edad de los consultantes era menor, en tanto que, por el contrario, las carencias y la vulnerabilidad económica y la pérdida de personas cercanas fueron significativamente más altas cuanto mayor era la edad. 
Tabla 3

Condiciones de vida y problemática psicosocial y de salud mental reportada por consultantes del servicio de atención telefónica Línea de la Vida, derivados a Centros de Integración Juvenil durante la epidemia de COVID-19

Diferencias significativas por grupo de edad

\begin{tabular}{|c|c|c|c|c|c|c|c|}
\hline & \multicolumn{2}{|c|}{$\begin{array}{l}\text { Adultos jóvenes } \\
\quad(n=398)\end{array}$} & \multicolumn{2}{|c|}{$\begin{array}{c}\text { Adultos } \\
(n=1,826)\end{array}$} & \multicolumn{2}{|c|}{$\begin{array}{l}\text { Adultos mayores } \\
\qquad(n=179)\end{array}$} & \multirow{2}{*}{$X^{2}, p(g l=2)$} \\
\hline & Frec. & $\%$ & Frec. & $\%$ & Frec. & $\%$ & \\
\hline \multicolumn{8}{|l|}{ Sexo } \\
\hline Hombres & 214 & 53.8 & 882 & 48.2 & 78 & 43.6 & \multirow{2}{*}{$6.110, .047$} \\
\hline Mujeres & 184 & 46.2 & 946 & 51.8 & 101 & 56.4 & \\
\hline \multicolumn{8}{|l|}{ Cohabitación durante la pandemia } \\
\hline El (la) consultante vive solo/a & 44 & 11.1 & 338 & 18.6 & 41 & 23.0 & $16.348,<.001$ \\
\hline Vive con su cónyuge o pareja & 141 & 35.4 & 1,016 & 55.6 & 95 & 53.1 & $53.266,<.001$ \\
\hline Vive con hijos jóvenes o adolescentes & 50 & 12.6 & 666 & 36.4 & 31 & 17.3 & $104.027,<.001$ \\
\hline Vive con niños & 128 & 32.2 & 524 & 28.7 & 14 & 7.8 & $40.131,<.001$ \\
\hline Vive con adultos mayores & 196 & 49.4 & 478 & 26.2 & 42 & 23.5 & $87.676,<.001$ \\
\hline \multicolumn{8}{|l|}{ Variables relacionadas con la pandemia } \\
\hline $\begin{array}{l}\text { El (la) consultante reporta síntomas de } \\
\text { COVID-19 }\end{array}$ & 139 & 34.9 & 526 & 28.8 & 46 & 25.7 & $7.276, .026$ \\
\hline Pertenece a un grupo vulnerable & 52 & 13.1 & 561 & 30.7 & 168 & 93.9 & $378.572,<.001$ \\
\hline Registra trastornos comórbiles de alto riesgo & 37 & 9.3 & 379 & 20.7 & 79 & 44.1 & $91.781,<.001$ \\
\hline $\begin{array}{l}\text { Durante la epidemia le ha sido posible en } \\
\text { general quedarse en casa }\end{array}$ & 242 & 61.0 & 1,201 & 65.7 & 130 & 72.6 & $7.687, .021$ \\
\hline \multicolumn{8}{|l|}{ Diagnóstico presuntivo de salud mental } \\
\hline Conducta violenta & 16 & 4.0 & 41 & 2.2 & 8 & 4.5 & $6.221, .045$ \\
\hline Conducta suicida & 20 & 5.0 & 25 & 1.4 & 6 & 3.4 & $22.478,<.001$ \\
\hline Abuso de sustancias & 25 & 6.3 & 45 & 2.5 & 6 & 3.4 & $15.606,<.001$ \\
\hline \multicolumn{8}{|l|}{ Otras problemáticas } \\
\hline $\begin{array}{l}\text { Enfrenta problemas económicos o necesidades } \\
\text { insatisfechas }\end{array}$ & 103 & 25.9 & 576 & 31.5 & 78 & 43.6 & $17.930,<.001$ \\
\hline $\begin{array}{l}\text { Ha experimentado dificultades en el cuidado } \\
\text { de niños pequeños }\end{array}$ & 39 & 9.8 & 138 & 7.6 & 4 & 2.2 & $10.072, .006$ \\
\hline $\begin{array}{l}\text { Ha sufrido la pérdida de una persona } \\
\text { cercana }\end{array}$ & 50 & 12.6 & 278 & 15.2 & 44 & 24.6 & $14.022, .001$ \\
\hline
\end{tabular}

\section{Comparación por lapso en el que se solicitó apoyo (Tabla 4)}

El análisis comparativo con base en el lapso en el que se solicitó apoyo refleja en primer lugar, un porcentaje significativamente más alto de casos que vivían con sus cónyuges, así como con adolescentes y niños pequeños, entre quienes consultaron en el periodo intermedio del 29 de junio al 26 de julio.

Entre el primer y tercer periodo se aprecia un aumento significativo de casos que reportaron síntomas de COVID-19; de igual modo, en los dos últimos lapsos un porcentaje mayor de consultantes habían convivido con personas con diagnóstico positivo, mientras que la proporción de consultantes que habían podido mantener el confinamiento doméstico tendió a disminuir.

Al inicio del lapso, se reportaron tasas relativamente mayores de conducta violenta y de abuso de sustancias, así como un porcentaje ligeramente más alto de personas que habían sido o temían ser blanco de violencia en el hogar, mientras que en el último de los lapsos se aprecia una proporción significativamente más alta de casos con síntomas depresivos, los dos últimos periodos comprenden un número más alto de casos que se habían contagiado o temían haberse contagiado. 
Finalmente, los casos que reportaron estados de intranquilidad y temor se concentraron con mayor frecuencia en los periodos intermedios, en tanto que en el último mes de seguimiento destaca un aumento significativo de consultantes que refirieron tristeza y depresión.

Tabla 4

Condiciones de vida y problemática psicosocial y de salud mental reportada por consultantes del servicio de atención telefónica Línea de la Vida, derivados a Centros de Integración Juvenil durante la epidemia de COVID-19

Diferencias significativas por fecha de consulta

\begin{tabular}{|c|c|c|c|c|c|c|c|c|c|}
\hline & \multicolumn{2}{|c|}{$\begin{array}{c}\text { Casos atendidos } \\
\text { del } \mathbf{1 1} \text { al } \\
\mathbf{3 1} \text { de mayo } \\
(n=665)\end{array}$} & \multicolumn{2}{|c|}{$\begin{array}{c}\text { Casos } \\
\text { atendidos del } \\
\mathbf{1} \text { al } \mathbf{2 8} \text { de junio } \\
(n=752)\end{array}$} & \multicolumn{2}{|c|}{$\begin{array}{c}\text { Casos } \\
\text { atendidos del } \\
29 \text { de junio al } \\
26 \text { de julio } \\
(n=632)\end{array}$} & \multicolumn{2}{|c|}{$\begin{array}{l}\text { Casos atendidos } \\
\text { del } 27 \text { de julio al } \\
23 \text { de agosto } \\
(n=354)\end{array}$} & \multirow[t]{2}{*}{$X^{2}, p(g l=3)$} \\
\hline & Frec. & $\%$ & Frec. & $\%$ & Frec. & $\%$ & Frec. & $\%$ & \\
\hline \multicolumn{10}{|l|}{ Cohabitación durante la pandemia } \\
\hline $\begin{array}{l}\text { El (la) consultante vive con su } \\
\text { cónyuge o pareja }\end{array}$ & 314 & 47.1 & 379 & 50.4 & 368 & 58.1 & 191 & 54.0 & $17.144, .001$ \\
\hline $\begin{array}{l}\text { Vive con hijos jóvenes o } \\
\text { adolescentes }\end{array}$ & 178 & 26.7 & 244 & 32.4 & 221 & 34.9 & 104 & 29.4 & $11.372, .010$ \\
\hline Vive con niños & 165 & 24.8 & 210 & 27.9 & 210 & 33.2 & 81 & 22.9 & $16.447, .001$ \\
\hline \multicolumn{10}{|l|}{$\begin{array}{l}\text { Variables relacionadas con la } \\
\text { pandemia }\end{array}$} \\
\hline $\begin{array}{l}\text { El (la) consultante reporta } \\
\text { síntomas de COVID-19 }\end{array}$ & 170 & 25.6 & 202 & 26.9 & 224 & 35.4 & 115 & 32.5 & $19.678,<.001$ \\
\hline $\begin{array}{l}\text { Ha convivido con alguien con } \\
\text { COVID-19 }\end{array}$ & 153 & 23.0 & 219 & 29.1 & 212 & 33.6 & 120 & 33.9 & $22.122,<.001$ \\
\hline $\begin{array}{l}\text { Registra trastornos comórbiles } \\
\text { de alto riesgo }\end{array}$ & 162 & 24.3 & 141 & 18.8 & 116 & 18.3 & 76 & 21.5 & $9.393, .025$ \\
\hline $\begin{array}{l}\text { Durante la epidemia le ha sido } \\
\text { posible en general quedarse } \\
\text { en casa }\end{array}$ & 471 & 70.7 & 493 & 65.6 & 407 & 64.4 & 202 & 57.2 & $19.061,<.001$ \\
\hline \multicolumn{10}{|l|}{$\begin{array}{l}\text { Diagnóstico presuntivo de salud } \\
\text { mental }\end{array}$} \\
\hline Depresión & 112 & 16.8 & 101 & 13.4 & 78 & 12.3 & 75 & 21.2 & $1.069, .001$ \\
\hline Conducta violenta & 32 & 4.8 & 18 & 2.4 & 8 & 1.3 & 7 & 2.0 & $17.156, .001$ \\
\hline Abuso de sustancias & 34 & 5.1 & 21 & 2.8 & 10 & 1.6 & 11 & 3.1 & $13.734, .003$ \\
\hline \multicolumn{10}{|l|}{ Otras problemáticas } \\
\hline $\begin{array}{l}\text { Ha sido o teme ser víctima de } \\
\text { violencia doméstica }\end{array}$ & 52 & 7.8 & 41 & 5.5 & 22 & 3.5 & 19 & 5.4 & $11.665, .009$ \\
\hline $\begin{array}{l}\text { Se ha contagiado o teme haberse } \\
\text { contagiado de COVID-19 }\end{array}$ & 327 & 49.1 & 431 & 57.3 & 400 & 63.2 & 219 & 61.9 & $30.290,<.001$ \\
\hline \multicolumn{10}{|l|}{$\begin{array}{l}\text { Estado de ánimo en la última } \\
\text { semana }\end{array}$} \\
\hline Intranquilo(a), con temor & 317 & 47.6 & 395 & 52.5 & 337 & 53.2 & 155 & 43.8 & $11.579, .009$ \\
\hline Triste, deprimido(a) & 69 & 10.4 & 70 & 9.3 & 45 & 7.1 & 44 & 12.4 & $8.362, .039$ \\
\hline
\end{tabular}




\section{Árbol de decisión (Figura I)}

El análisis de segmentación arrojó cuatro predictores significativos del diagnóstico de ansiedad o estrés: en primer lugar, problemas en el cuidado de familiares enfermos, con discapacidad o de la tercera edad $\left(X^{2}=58.744\right.$, $p<.001, g l=1)$; en un segundo nivel, en sentido descendente, la pérdida de personas cercanas $\left(X^{2}=29.178\right.$, $p<.001, g l=1)$ y el contacto con alguna persona enferma $\left(X^{2}=12.484, p<.001, g /=1\right)$ y finalmente, en un tercer nivel, el contagio o el temor de haberse contagiado de COVID-19 $\left(X^{2}=9.321, p=.001, g l=1\right)$. El árbol obtenido se compuso de nueve nodos, cinco de ellos terminales (nodos 4, 5, 6, 7 y 8), con un riesgo estimado de 0.324 (error típico de .01). Incluido en el análisis, el consumo de sustancias no figuró entre los predictores significativos de ansiedad o estrés, lo mismo que otras variables como edad, sexo, lapso de consulta, problemas económicos, violencia doméstica, vulnerabilidad o síntomas de COVID-19 y apego al confinamiento doméstico.

En el árbol destacan, en primer lugar, los nodos situados en el extremo inferior de la rama izquierda (nodos 7 y 8) que, en conjunto, reúnen una alta proporción de casos ( $76.1 \%$ del total) y que, además, presentan un porcentaje relativamente elevado de casos diagnosticados con ansiedad o estrés. El nodo 7 agrupa personas que, a pesar de no haber reportado ninguna de las variables independientes incluidas en el análisis, refirieron síntomas de ansiedad o estrés en $59.2 \%$; en tanto que el nodo 8 comprende casos que se habrían contagiado de COVID-19 o temerían haberlo hecho, con una prevalencia de síntomas de ansiedad o estrés de 65.4\%. En la misma rama izquierda del árbol, en el nivel intermedio, se localiza el nodo terminal 4 que integra $10.7 \%$ de los casos de la muestra, con un alto porcentaje de casos de ansiedad o estrés (79.8\%) que habrían experimentado la pérdida de una persona cercana.

En el nivel intermedio de la rama derecha del árbol, se aprecian dos nodos terminales (nodos 5 y 6) que, si bien comprenden un menor número de casos $(6.9 \%$ y $6.4 \%$ de la muestra, respectivamente), registran porcentajes de casos diagnosticados con ansiedad o estrés notoriamente altos ( $92.7 \%$ y $79.7 \%$, respectivamente). Ambos nodos comprenden casos que reportaron haber tenido dificultades en el cuidado de familiares enfermos, a lo cual se suman, en el caso del nodo 5 , casos que habrían tenido contacto con personas enfermas de COVID-19.

\section{DISCUSIÓN}

En concordancia con lo reportado en diversos estudios realizados en el mundo (Hyland et al., 2020; Özdin
\& Bayrak Özdin, 2020; Qiu et al., 2020; Rehman et al., 2020; Verma \& Mishra, 2020; Wong et al., 2020, entre otros), los resultados expuestos identifican a la ansiedad o estrés, así como a la depresión, como los trastornos de salud mental más frecuentes entre los consultantes del servicio Línea de la Vida derivados a ClJ.

Sin embargo, tal como se ha expuesto, los problemas de salud, sociales, interpersonales y de salud mental derivados de la contingencia sanitaria por la pandemia, reflejan diferencias atribuibles al sexo, la edad de la población y el desarrollo de la epidemia. De este modo, se aportan algunos elementos para identificar condiciones y factores que parecen haber incidido en la problemática reportada por la población consultante durante la etapa más aguda de la epidemia en México.

Así, entre estos factores se puede referir, en primer lugar, condiciones y factores relacionados con la construcción social del género, tales como la adscripción social de las mujeres al espacio doméstico-familiar y a tareas del cuidado informal de los hijos, enfermos y adultos mayores (Jiménez \& Moya, 2017). Si bien es probable que por esta razón las mujeres refieran haber podido respetar con mayor frecuencia el confinamiento doméstico durante la epidemia, se verían también mayormente sometidas a dificultades desbordantes relacionadas con la asignación de estas funciones, agravadas por el aumento de la carga de trabajo no remunerado durante la crisis, al tiempo que, con frecuencia, debían continuar realizando actividades laborales remuneradas (Cantillo et al., 2018; Saban \& Barone, 2020; Tello \& Vargas, 2020).

Asimismo, debe destacarse que las mujeres se reconocieron en una mayor proporción como población vulnerable, refiriendo una mayor exposición a la violencia doméstica, así como a índices más altos de trastornos afectivos (ansiedad, estrés y depresión), como lo reportan otros estudios (Lin et al., 2020; Mazza, De Lorenzo et al., 2020; Özdin \& Bayrak Özdin, 2020; Pappa, 2020; Qu et al., 2020; Savitsky et al., 2020; Wong et al., 2020).

Por su parte, los hombres refirieron con mayor frecuencia síntomas relacionados con COVID-19, lo cual podría estar relacionado con una mayor dificultad para mantener el confinamiento y una mayor exposición al contagio en espacios públicos, dado el encargo social hegemónico de hacerse cargo de las actividades económicas para proveer a la familia. Además, presentaron más alto consumo de sustancias, lo cual concuerda con los datos reportados por múltiples encuestas y sistemas de información (Gutiérrez, 2020; Instituto Nacional de Psiquiatría Ramón de la Fuente Muñiz [INPRFM], Instituto Nacional de Salud Pública [INSP], Comisión Nacional contra las Adicciones [CONADIC], Secretaría de Salud [SSA], 2017; United Nations Office on Drugs and Crime [UNODC], 2020). 
Figura 1

Árbol de decisión. Segmentación de la muestra de acuerdo con predictores significativos del diagnóstico presuntivo de ansiedad/estrés

\section{Diagnóstico presuntivo de ansiedad/estrés}

\section{Con ansiedad/estrés}

Sin ansiedad/estrés

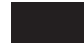

\begin{tabular}{ccc}
\multicolumn{3}{c}{ NODO 0 } \\
\hline Categoría & $\%$ & $\mathbf{n}$ \\
\hline Con ansiedad & 67.6 & 1625
\end{tabular}

\begin{tabular}{ccc} 
Sin ansiedad & 32.4 & 780 \\
\hline Total & 100.00 & 2405 \\
\hline & & \\
\hline & & \\
\hline & & \\
\hline & & \\
\hline
\end{tabular}

Problemas en el cuidado de familiares $\chi^{2}=58.744, g l=1, p<.001$

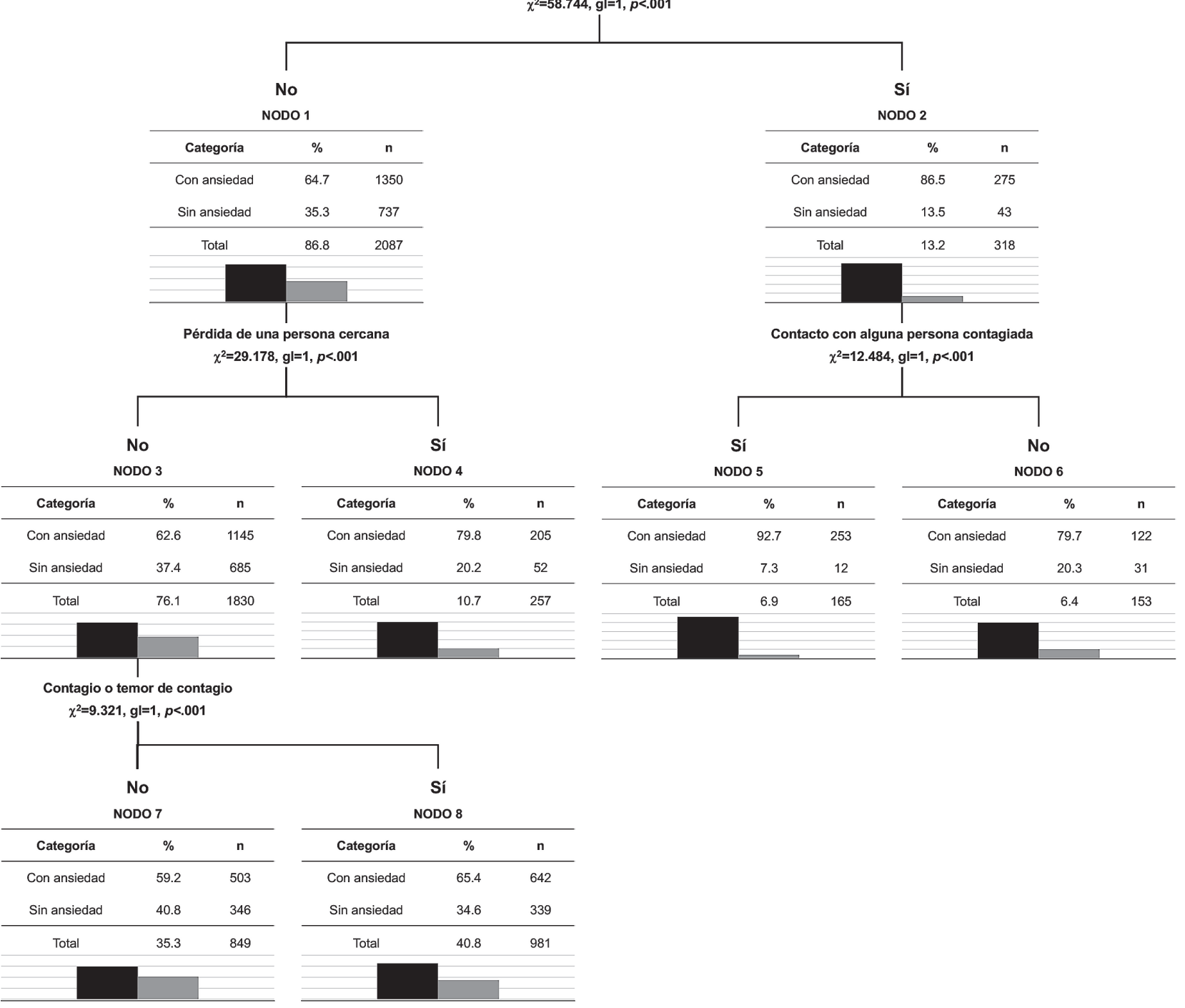


En segundo sitio, el estudio documenta diversas condiciones que pueden estar vinculadas con el ciclo de vida de las personas, manifiestas, en el caso de adultos mayores, en una mayor vulnerabilidad y un más alto riesgo frente al COVID-19 (Lithander et al., 2020; Mueller et al., 2020; Shahid et al., 2020), un mayor aislamiento social $y$, probablemente, una menor movilidad relacionada con una mayor posibilidad de respetar el confinamiento doméstico, pero también con una mayor vulnerabilidad económica (Dickens et al., 2011; Gajardo, 2015), a su vez con más frecuentes pérdidas cercanas que, sin embargo, no necesariamente se traducen en mayores tasas de depresión que en otros grupo etarios (Gamo \& Pazos, 2009).

En contraste, en razón de su posición vital, el grupo de menor edad (jóvenes adultos) se vería afectado en mayor medida por dificultades en el cuidado y crianza de niños pequeños pero, igualmente, por el abuso de sustancias y conductas violentas o suicidas, trastornos de alta prevalencia en adultos jóvenes (Borges et al., 2010; INPRFM, INSP, CONADIC, SS, 2017; Miron et al., 2019; Rodríguez et al., 2006; Valdez et al., 2013), así como por una mayor exposición al contagio, reportando una mayor prevalencia de síntomas de COVID-19, lo cual podría ser atribuible a la percepción de un menor riesgo de desarrollar los síntomas graves de la enfermedad, así como a un menor apego a las medidas adoptadas de reducción de la movilidad (Mueller et al., 2020; Nivette et al., 2020).

Por lo que se refiere a la comparación realizada en el lapso en que se llevó a cabo la consulta, los hallazgos reflejan el aumento de factores atribuibles al avance mismo de la epidemia en el país (SSA, 2020), tales como un incremento de casos con síntomas de COVID-19 y un contacto más frecuente con personas que padecen la enfermedad, aspectos que también podrían relacionarse con el incremento gradual de la movilidad y el retorno a actividades económicas, con la consecuente disminución del confinamiento, lo cual puede vincularse con el aumento del desempleo y otros problemas económicos, tal como refieren los propios consultantes y como se reporta en otros estudios (Jiménez-Bandala et al., 2020; Samaniego, 2020). Cabe subrayar el incremento de estados de intranquilidad y de tristeza y, en particular, de presuntas formas clínicas de la depresión; lo mismo que la tendencia creciente del temor a haberse contagiado.

Finalmente, los resultados del análisis de segmentación muestran el alto peso que se atribuye a los efectos inmediatos de la epidemia, explícitamente la convivencia y contacto con enfermos, los problemas en el cuidado de éstos, las pérdidas cercanas y el contagio o riesgo de ello, en la configuración de una problemática de salud mental en la que sobresalen de manera preponderante los trastornos de ansiedad y estrés (Ni et al., 2020; Özdin \& Bayrak, 2020).
En cuanto al alcance y limitaciones del presente estudio, si bien destaca su aportación para identificar y describir la problemática psicosocial y de salud mental que ha aquejado a la población mexicana en el curso de la epidemia de COVID-19, no obstante deben señalarse condiciones como la no representatividad de la muestra, la selección parcial de indicadores dada la coyuntura de premura en que se levantó la información, las mediciones de tipo categórico diseñadas para facilitar la toma de datos en una situación de urgencia psicológica orientada primariamente a otros fines, distintos de la investigación y, finalmente, la falta de acceso a la fuente primaria de información y su registro indirecto por parte de los operadores del servicio.

\section{AGRADECIMIENTOS:}

Los autores agradecen la contribución del doctor Héctor Francisco Gómez Estrada, la psicóloga Rocío Romero Reséndez y el doctor Víctor Manuel Guisa Sánchez, quienes coordinaron operativamente la atención a consultantes y llevaron a cabo la integración de bases de datos.

\section{FUENTES DE FINANCIAMIENTO}

Este estudio no contó con fuentes de financiamiento.

\section{CONFLICTOS DE INTERÉS}

No existe ningún conflicto de interés en la realización de este artículo.

\section{REFERENCIAS}

Abad, C., Fearday, A., \& Safdar, N. (2010). Adverse effects of isolation in hospitalised patients: a systematic review. The Journal of hospital infection, 76(2), 97-102. doi: 10.1016/j. jhin.2010.04.027

Active Minds. (Abril de 2020). COVID-19 impact on college student mental health. https://www.activeminds.org/studentsurvey/

Anurudran, A., Yared, L., Comrie, C., Harrison, K., \& Burke, T. (2020). Domestic violence amid COVID-19. International Journal of Gynecology and Obstetrics, 150(2), 255-256. doi: 10.1002/ijgo.13247

Borges, G., Orozco, R., Benjet, C., \& Medina-Mora, M. E. (2010). Suicidio y conductas suicidas en México: Retrospectiva y situación actual. Salud Pública de México, 52(4), 292-304.

Brooks, S. K., Webster, R. K., Smith, L. E., Woodland, L., Wessely, S., Greenberg, N., \& Rubin, G. J. (2020). The psychological impact of quarantine and how to reduce it: Rapid review of the evidence. The Lancet, 395(10227), 912-920. doi:10.1016/s01406736(20)30460-8

Bruine de Bruin, W. (2020). Age Differences in COVID-19, Risk Perceptions and Mental Health: Evidence From a National U.S. 
Survey Conducted in March 2020. The Journals of Gerontology: Series B, 1-6. Doi: 10.1093/geronb/gbaa074

Cantillo, M., Lleopart, T., \& Ezquerra, S. (2018). El cuidado informal en tiempos de crisis. Análisis desde la perspectiva enfermera. Enfermería Global, 17(2), 515-541. doi: 10.6018/eglobal.17.2.297211

Cluver, L., Lachman, J. M., Sherr, L., Wessels, I., Krug, E., Rakotomalala, S., Blight, S., Hillis, S., Bachman, G., Green, O., Butchart, A., Tomlinson, M., Ward, C. L., Doubt, J., \& McDonald, K. (2020). Parenting in a time of COVID-19. Lancet, 395(10231), e64. doi: 10.1016/S0140-6736(20)30736-4

Day, H., Perencevich, E., Harris, A., Gruber-Baldini, A., Himelhoch, S., Brown, C., \& Morgan, D. (2013). Depression, Anxiety, and Moods of Hospitalized Patients under Contact Precautions. Infection Control \& Hospital Epidemiology, 34(3), 251-258. doi:10.1086/669526

Del Río Lozano, M., \& García Calvente, M. del M. (2020). Cuidados y abordaje de la pandemia de COVID-19 con enfoque de género. Gaceta Sanitaria. doi: 10.1016/j.gaceta.2020.05.006

Dickens, A., Richards, S., Greaves, C., \& Campbell, J. (2011). Interventions targeting social isolation in older people: A systematic review. BMC Public Health 11, 647. doi: 10.1186/14712458-11-647

DiGiovanni, C., Conley, J., Chiu, D., \& Zaborski, J. (2004). Factors influencing compliance with quarantine in Toronto during the 2003 SARS outbreak. Biosecurity and bioterrorism: biodefense strategy, practice, and science, 2(4), 265-272. doi: 10.1089/ bsp.2004.2.265

Durankuş, F., \& Aksu, E. (2020). Effects of the COVID-19 pandemic on anxiety and depressive symptoms in pregnant women: A preliminary study. Journal of Maternal-Fetal and Neonatal Medicine, 1-7. doi: 10.1080/14767058.2020.1763946

Elbay, R. Y., Kurtulmuş, A., Arpacıoğlu, S., \& Karadere, E. (2020). Depression, anxiety, stress levels of physicians and associated factors in COVID-19 pandemics. Psychiatry Research, 290, 113130. doi: 10.1016/j.psychres.2020.113130

Fitzpatrick, K. M., Harris, C., \& Drawve, G. (2020). Fear of COVID-19 and the mental health consequences in America. Psychological Trauma: Theory, Research, Practice, and Policy, 12, 17-21. doi: 10.1037/tra0000924

Gajardo, J. (2015). Vejez y soledad: Implicancias a partir de la construcción de la noción de riesgo. Acta Bioethica, 21(2), 199-205.

Gamo, E., \& Pazos, P. (2009). El duelo y las etapas de la vida. Revista de la Asociación Española de Neuropsiquiatría, 29(104), 455-469.

Gobierno de México. (2020) Conferencias de Prensa. https://coronavirus.gob.mx/category/conferencias-de-prensa/

Gulati, G., \& Kelly, B. D. (2020). Domestic violence against women and the COVID-19 pandemic: What is the role of psychiatry? International Journal of Law and Psychiatry, 71, 101594. doi: 10.1016/j.ijlp.2020.101594

Gutiérrez, A. D. (2020). Consumo de drogas en solicitantes de tratamiento en Centros de Integración Juvenil, Drogas ilícitas, Primer semestre de 2020. (Informe de investigación 20-09a). Centros de Integración Juvenil. http://www.cij.gob.mx/programas/Investigacion/Epidemiologica.asp

Hawryluck, L., Gold, W. L., Robinson, S., Pogorski, S., Galea, S., \& Styra, R. (2004). SARS control and psychological effects of quarantine, Toronto, Canada. Emerging infectious diseases, 10(7), 1206-1212. doi: 10.3201/eid1007.030703

Huarcaya-Victoria, J. (2020). Consideraciones sobre la salud mental en la pandemia de COVID-19. Revista Peruana de Medicina EXperimental y Salud Pública, 37(2), 327-334

Humphreys, K. L., Myint, M. T., \& Zeanah, C. H. (2020). Increased Risk for Family Violence During the COVID-19 Pandemic. Pediatrics, 146(1), e20200982. doi: 10.1542/peds.2020-0982

Huremović, D. (Ed.). (2019). Psychiatry of pandemics. A mental health response to infection outbreak. Springer Nature. doi: 10.1007/9783-030-15346-5

Hyland, P., Shevlin, M., McBride, O., Murphy, J., Karatzias, T., Bentall, R. P., Martinez, A., \& Vallières, F. (2020). Anxiety and depression in the Republic of Ireland during the COVID-19 pandemic. Acta Psychiatrica Scandinavica, 142(3), 249-256. doi: 10.1111/acps.13219

Instituto Nacional de Psiquiatría Ramón de la Fuente Muñiz, Instituto Nacional de Salud Pública, Comisión Nacional contra las Adicciones, Secretaría de Salud. (2017). Encuesta Nacional de Consumo de Drogas, Alcohol y Tabaco 2016-2017: Reporte de Drogas. INPRFM.

Jiménez, I., \& Moya, M. (2017). The family caregiver: The naturalized sense of obligation in women to be caregivers. Enfermería Global, 17(1), 420-447. doi: 10.6018/eglobal.17.1.292331

Jiménez-Bandala, C., Peralta, J., Sánchez, E., Márquez, I., \& Arellano, D. (2020). La situación del mercado laboral en México antes y durante la COVID-19. Revista Internacional de Salarios Dignos, 2(2), 1-9.

Johal S. S. (2009). Psychosocial impacts of quarantine during disease outbreaks and interventions that may help to relieve strain. The New Zealand Medical Journal, 122(1296), 47-52.

Johnson, M. C., Saletti-Cuesta, L., \& Tumas, N. (2020). Emociones, preocupaciones y reflexiones frente a la pandemia del COVID-19 en Argentina. Ciência \& Saúde Coletiva, 25(supl.1), 2447-2456.

Jungmann, S. M., \& Witthöft, M. (2020). Health anxiety, cyberchondria, and coping in the current COVID-19 pandemic: Which factors are related to coronavirus anxiety? Journal of Anxiety Disorders, 73, 102239. doi: 10.1016/j.janxdis.2020.102239

Karaşar, B., \& Canli, D. (2020). Psychological resilience and depression during the COVID-19 pandemic in Turkey. Psychiatria Danubina, 32(2), 273-279. doi: 10.24869/psyd.2020.273

Li, W., Yang, Y., Liu, Z. H., Zhao, Y. J., Zhang, Q., Zhang, L., Cheung, T., \& Xiang, Y. T. (2020). Progression of mental health services during the COVID-19 outbreak in China. International Journal of Biological Sciences, 16(10), 1732-1738. doi: 10.7150/ijbs.45120

Lin, Y., Hu, Z., Alias, H., \& Wong, L. P. (2020). Knowledge, Attitudes, Impact, and Anxiety Regarding COVID-19 Infection Among the Public in China. Frontiers in Public Health, 8, 236. doi: 10.3389/ fpubh.2020.00236

Lithander, F. E., Neumann, S., Tenison, E., Lloyd, K., Welsh, T. J., Rodrigues, J., Higgins, J., Scourfield, L., Christensen, H., Haunton, 
V. J., \& Henderson, E. J. (2020). COVID-19 in older people: a rapid clinical review. Age and ageing, 49(4), 501-515. doi: 10.1093/ ageing/afaa093

Luo, M., Guo, L., Yu, M., \& Wang, H. (2020). The psychological and mental impact of coronavirus disease 2019 (COVID-19) on medical staff and general public, A systematic review and meta-analysis. Psychiatry Research, 291, 113190. doi: 10.1016/j. psychres.2020.113190

Martínez-Taboas, A. (2020). Pandemias, COVID-19 y salud mental: ¿Qué sabemos actualmente? Revista Caribeña de Psicología, 4(2), 143-152. doi: 10.37226/rcp.v4i2.4907

Mazza, M., De Lorenzo, R., Conte, C., Poletti, S., Vai, B., Bollettini, I., Melloni, E. M. T., Furlan, R., Ciceri, F., Rovere-Querini, P., \& Benedetti, F. (2020). Anxiety and depression in COVID-19 survivors: Role of inflammatory and clinical predictors. Brain, Behavior, and Immunity. doi: 10.1016/j.bbi.2020.07.037

Mazza, M., Marano, G., Lai, C., Janiri, L., \& Sani, G. (2020). Danger in danger: Interpersonal violence during COVID-19 quarantine. Psychiatry research, 289, 113046. doi: 10.1016/j.psychres.2020.113046

McMahon, S. A., Ho, L. S., Brown, H., Miller, L., Ansumana, R., \& Kennedy, C. E. (2016) Healthcare providers on the frontlines: a qualitative investigation of the social and emotional impact of delivering health services during Sierra Leone's Ebola epidemic. Health Policy and Planning, 31(9), 1232-1239. doi: 10.1093/heapol/czw055

Mental Health America. (2020). COVID-19 and mental health: What we are learning from www.mhascreening.org. July 1, 2020 Recuperado de: https://mhanational.org/sites/default/files/Coronavirus Mental Health Presentation 7-1-2020.pdf

Miron, O., Yu, K. H., Wilf-Miron, R., \& Kohane, I. S. (2019). Suicide rates among adolescents and young adults in the United States, 20002017. JAMA, 321(23), 2362-2364. doi: 10.1001/jama.2019.5054

Mosheva, M., Hertz-Palmor, N., Dorman Ilan, S., Matalon, N., Pessach, I. M., Afek, A., Ziv, A., Kreiss, Y., Gross, R., \& Gothelf, D. (2020). Anxiety, pandemic-related stress and resilience among physicians during the COVID-19 pandemic. Depression and Anxiety, 37(10), 965-971. doi: 10.1002/da.23085

Mueller, A. L., McNamara, M. S., \& Sinclair, D. A. (2020). Why does COVID-19 disproportionately affect older people? Aging, 12(10), 9959-9981. doi: 10.18632/aging.103344

Ni, M. Y., Yang, L., Leung, C. M. C., Li, N., Yao, X. I., Wang, Y., Leung, G. M., Cowling, B. J., \& Liao, Q. (2020). Mental health, risk factors, and social media use during the COVID-19 epidemic and cordon sanitaire among the community and health professionals in Wuhan, China: Cross-sectional survey. JMIR Mental Health, 7(5), e19009. doi: 10.2196/19009

Nivette, A., Ribeaud, D., Murray, A., Steinhoff, A., Bechtiger, L., Hepp, U., Shanahan, L., \& Eisner, M. (2020). Non-compliance with COVID-19-related public health measures among young adults in Switzerland: Insights from a longitudinal cohort study. Social Science \& Medicine, 268, 113370. doi: 0.1016/j.socscimed.2020.113370
O'Leary, A., Jalloh, M. F., \& Neria, Y. (2018). Fear and culture: Contextualising mental health impact of the 2014-2016 Ebola epidemic in West Africa. BMJ global health, 3(3), e000924. doi: 10.1136/bmjgh-2018-000924

Organización de Estados Americanos-Comisión Interamericana de Mujeres. (2020). COVID-19 en la vida de las mujeres. Razones para reconocer los impactos diferenciados. Recuperado de https://www.oas.org/es/cim/docs/ArgumentarioCOVID19-ES.pdf

Organización Mundial de la Salud. (2019). Brote de enfermedad por coronavirus (COVID-19): orientaciones para el público. Recuperado de https://www.who.int/es/emergencies/diseases/novel-coronavirus-2019/advice-for-public

Ozamiz-Etxebarria, N., Dosil-Santamaria, M., Picaza-Gorrochategui, M., \& Idoiaga-Mondragon, N. (2020). Stress, anxiety, and depression levels in the initial stage of the COVID-19 outbreak in a population sample in the northern Spain. Cadernos de Saude Publica, 36(4), 1-10. doi: 0.1590/0102-311X00054020

Özdin, S., \& Bayrak Özdin, Ş. (2020). Levels and predictors of anxiety, depression and health anxiety during COVID-19 pandemic in Turkish society: The importance of gender. International Journal of Social Psychiatry, 66(5), 504-511. doi: 10.1177/0020764020927051

Pappa, S., Ntella, V., Giannakas, T., Giannakoulis, V. G., Papoutsi, E., \& Katsaounou, P. (2020). Prevalence of depression, anxiety, and insomnia among healthcare workers during the COVID-19 pandemic: A systematic review and meta-analysis. Brain, Behavior, and Immunity, 88, 901-907. doi: 10.1016/j. bbi.2020.05.026

Qiu, J., Shen, B., Zhao, M., Wang, Z., Xie, B., \& Xu, Y. (2020). A nationwide survey of psychological distress among Chinese people in the COVID-19 epidemic: Implications and policy recommendations. General Psychiatry, 33(2), 19-21. doi: 10.1136/ gpsych-2020-100213

Rajkumar, R. P. (2020). COVID-19 and mental health: A review of the existing literature. Asian Journal of Psychiatry, 52, 102066. doi: 10.1016/j.ajp.2020.102066

Ramírez-Ortiz, J., Castro-Quintero, D., Lerma-Córdoba, C., Yela-Ceballos, F., \& Escobar-Córdoba, F. (2020). Consecuencias de la pandemia COVID-19 en la salud mental asociadas al aislamiento social. Revista Colombiana de Anestesiología, 48(4), e930. 10.5554/22562087.e930

Rehman, U., Shahnawaz, M. G., Khan, N. H., Kharshiing, K. D., Khursheed, M., Gupta, K., Kashyap, D., \& Uniyal, R. (2020). Depression, anxiety and stress among Indians in times of COVID-19 lockdown. Community Mental Health Journal. doi: 10.1007/ s10597-020-00664-x

Rodríguez, J., Fernández, A. M., Hernández, E., \& Ramírez, S. (2006) Conductas agresivas, consumo de drogas e intentos de suicidio en jóvenes universitarios. Terapia Psicológica, 24(1), 63-69.

Roesch, E., Amin, A., Gupta, J., \& García-Moreno, C. (2020). Violence against women during COVID-19 pandemic restrictions. BMJ, 369, m1712. doi: 10.1136/bmj.m1712

Saban, M., \& Barone, C. (2020) 100 días COVID: tareas de cuidado y productividad. Consejo Económico y Social de la 
Ciudad de Buenos Aires. http://bdigital.cesba.gob.ar/handle/123456789/467

Sacco, M. A., Caputo, F., Ricci, P., Sicilia, F., De Aloe, L., Bonetta, C. F., Cordasco, F., Scalise, C., Cacciatore, G., Zibetti, A., Gratteri, S., \& Aquila, I. (2020). The impact of the Covid-19 pandemic on domestic violence: The dark side of home isolation during quarantine. Medico-Legal Journal, 88(2), 71-73. doi: 10.1177/0025817220930553

Salari, N., Hosseinian-Far, A., Jalali, R., Vaisi-Raygani, A., Rasoulpoor, S., Mohammadi, M., Rasoulpoor, S., \& Khaledi-Paveh, B. (2020). Prevalence of stress, anxiety, depression among the general population during the COVID-19 pandemic: a systematic review and meta-analysis. Globalization and health, 16(1), 57. doi: 10.1186/s12992-020-00589-w

Samaniego, N. (2020). El COVID-19 y el desplome del empleo en México. Economía UNAM, 17(51). 306-314. doi: 10.22201/ fe.24488143e.2020.51.566

Savitsky, B., Findling, Y., Ereli, A., \& Hendel, T. (2020). Anxiety and coping strategies among nursing students during the COVID-19 pandemic. Nurse Education in Practice, 46, 102809. doi: 10.1016/j.nepr.2020.102809

Secretaría de Salud. (2020). Datos abiertos. Dirección General de Epidemiología. https://www.gob.mx/salud/documentos/datos-abiertos-152127

Shahid, Z., Kalayanamitra, R., McClafferty, B., Kepko, D., Ramgobin, D., Patel, R., Aggarwal, C. S., Vunnam, R., Sahu, N., Bhatt, D., Jones, K., Golamari, R., \& Jain, R. (2020). COVID-19 and Older Adults: What We Know. Journal of the American Geriatrics Society, 68(5), 926-929. doi: 10.1111/jgs. 16472
Tello de la Torre, C. \& Vargas Villamizar, O. H. (2020). Género y trabajo en tiempos del COVID-19: Una mirada desde la interseccionalidad. Revista Venezolana de Gerencia, 25(9), 389-393.

Torales, J., O'Higgins, M., Castaldelli-Maia, J. M., \& Ventriglio, A. (2020). The outbreak of COVID-19 coronavirus and its impact on global mental health. International Journal of Social Psychiatry, 66(4), 317-320. doi: 10.1177/0020764020915212

Tsamakis, K., Rizos, E., Manolis, A., Chaidou, S., KympouropouIos, S., Spartalis, E., Spandidos, D., Tsiptsios, D., \& TriantafyIlis, A. (2020). [Comment] COVID-19 pandemic and its impact on mental health of healthcare professionals. Experimental and Therapeutic Medicine, 19, 3451-3453. doi: 10.3892/ etm.2020.8646

United Nations Office on Drugs and Crime. (2020). Drug use and health consequences, World Drug Report 2020. World Health Organization.

Valdez-Santiago, R., Hidalgo-Solórzano, E., Mojarro-Íñiguez, M., Rivera-Rivera, L., \& Ramos-Lira, L. (2013). Violencia interpersonal en jóvenes mexicanos y oportunidades de prevención. Salud Pública de México, 55(Supl 2), S259-S266.

Verma, S., \& Mishra, A. (2020). Depression, anxiety, and stress and socio-demographic correlates among general Indian public during COVID-19. International Journal of Social Psychiatry, 66(8), 756-762, doi: 10.1177/0020764020934508

Wong, L. P., Hung, C. C., Alias, H., \& Lee, T. S. H. (2020). Anxiety symptoms and preventive measures during the COVID-19 outbreak in Taiwan. BMC Psychiatry, 20(1), 376. doi: 10.1186/ s12888-020-02786-8 